\title{
Educação Física e Atenção Primária à Saúde: o apoio matricial no contexto das redes
}

Physical Education and Primary Health Care: matrix support in health care networks context

\section{AUTORES \\ Braulio Nogueira de Oliveira ${ }^{1}$ (I) \\ Felipe Wachs ${ }^{2}$ (D) \\ ${ }^{1}$ Instituto Federal de Educação, Ciência e Tecnologia do Maranhão, Campus Avançado Porto \\ Franco, Porto Franco, Maranhão, Brasil. \\ ${ }^{2}$ Universidade Federal de Goiás, Faculdade de Educação Física e Dança, Goiânia, Goiás, Brasil.}

\section{CONTATO}

Braulio Nogueira de Oliveira

brauliono08@hotmail.com

Rua Custódio Barbosa, 09, Centro, Porto

Franco, Maranhão. Brasil.

CEP: 65970-000.

DOI

$10.12820 /$ rbafs. $23 \mathrm{e} 0064$

\begin{abstract}
RESUMO
O presente artigo problematiza o trabalho da Educação Física na Atenção Primária à Saúde (APS) com apoio matricial, na perspectiva das redes (setorial, intersetorial e social de apoio) e suas respectivas práticas desenvolvidas. A metodologia adotada foi qualitativa e as informações foram produzidas a partir de grupos focais e de observação da prática profissional. Os profissionais de Educação Física sinalizaram intervir com apoio matricial em cinco diferentes Redes de Atenção à Saúde (RAS) e indicaram desenvolver sete práticas através das quais exercem esse apoio matricial: apoio matricial intersetorial, acolhimento, projeto terapêutico singular, interconsulta, intervisita, apoio a unidades básicas de saúde e apoio a grupos de práticas corporais cuidados por líderes comunitários ou por agentes comunitários de saúde. Conclui-se que a complexidade dos cenários e as práticas de apoio matricial da Educação Física na APS, no contexto das RAS, revelam a limitação de se restringir a intervenção à prescrição de protocolos/procedimentos universais a partir de variáveis predeterminadas (de tempo e intensidade, por exemplo) por condição clínica. Aponta-se também para a necessidade de se considerar a estrutura operacional das redes (setorial, intersetorial e social de apoio), bem como as tecnologias/práticas centradas no trabalho interprofissional, pedagógico-formativo e centrado no usuário.
\end{abstract}

Palavras-chave: Atenção Primária à Saúde; Educação física e treinamento; Saúde da família; Apoio matricial; Redes de Atenção à Saúde.

\begin{abstract}
This paper discusses the intervention of the Physical Education professional at the Primary Health Care using a tool called matrix support, under a network perspective (sectoral, intersectoral and social support) and their practices. The methodology adopted was qualitative and the data was produced based on focal groups and observation of the professional practice. The participants showed to work with matrix support among five different Health Care Networks and indicated to develop seven practices through which they exercise the matrix support: intersectoral matrix support, "acolbimento", unique therapeutic project, "interconsulta", "intervisita", support to Primary Health Care services and support to bodily practices groups conducted by community leaders or community health agents. It is concluded that the complexity of the scenarios and the practices of matrix support made by Physical Education professional in Health Care Networks contexts reveal the limitation of restricting the intervention to prescribed universal protocols / procedures from predetermined variables (intensity, e.g.) by clinical condition. It is also pointed out the need to consider the operational structure of health care networks (sectoral, intersectoral and social support), as well as technologies or practices focused on interprofessional, educational and user-centered work.
\end{abstract}

Keywords: Primary Health Care; Physical Education and training; Family health; Matrix support; Health Care Networks.

\section{Introdução}

A Política Nacional de Atenção Básica (PNAB) tem na Saúde da Família a estratégia prioritária de expansão e consolidação da Atenção Básica ${ }^{1}$. Essa, por sua vez, constitui-se, majoritariamente, pelas equipes de Saúde da Família. Essas equipes contêm, no mínimo, profissionais auxiliares e/ou técnicos de enfermagem, de enfermagem, de medicina e entre quatro e doze agentes comunitários de saúde (ACS), podendo ser acrescidas por equipe de Saúde Bucal, com cirurgião-dentista, auxiliar e/ou técnico de Saúde Bucal ${ }^{1}$. Cada equipe de Saúde da Família se responsabiliza por uma área territorial, constituída a partir do cadastramento de famílias por ACS, produzindo microáreas ${ }^{1}$.

A Educação Física se insere no contexto da Atenção Primária à Saúde (APS) por meio, principalmente, dos Núcleos Ampliados de Saúde da Família - NASF-AB, conforme a $\mathrm{PNAB}^{1}$. Aos profissionais de Educação Física cabe atuar "diretamente no apoio matricial às equipes [de Saúde da Família] da(s) unidade(s) à(s) qual(is) o NASF está vinculado e no território destas equipes"2. Mesmo antes da criação do NASF, Ceccim \& Bilibio $^{3}$ já 
apontavam que "a inserção da educação física no Sistema Único de Saúde (SUS) passa pela introdução de seus saberes e práticas de maneira direta e/ou por meio do apoio matricial a ser prestado à equipe interprofissional".

Apesar de a revisão da PNAB promovida em 2017 tencionar as diretrizes propostas para os NASF, inicialmente, compreendemos que a função principal da Educação Física continua sendo "dar suporte (clínico, sanitário e pedagógico) aos profissionais das equipes de Saúde da Família (eSF) e de Atenção Básica (eAB)"1 e ao trabalho no Programa Academia da Saúde. A PNAB prevê que os profissionais do NASF "devem, a partir das demandas identificadas no trabalho conjunto com as equipes, atuar de forma integrada à Rede de Atenção à Saúde (RAS) e seus diversos pontos de atenção, além de outros equipamentos sociais públicos/ privados, redes sociais e comunitárias" ${ }^{1}$. O cenário das redes se torna ainda mais complexo quando se inserem nesse escopo a rede intersetorial e a rede social de apoio, particular de cada usuário.

Vale destacar que existem debates na área que envolve a organização dos serviços da Educação Física no NASF contextualizados com a proposta de trabalho fundamentado no apoio matricial ${ }^{4-8}$. Sem a pretensão de esgotar a discussão, o presente artigo objetiva analisar duas temáticas ainda pouco abordadas: a organização do trabalho da Educação Física na APS no contexto das redes, em particular o direcionamento da intervenção (público com o qual se envolve); e tecnologias/práticas do profissional de Educação Física no NASF centradas no usuário e fundamentadas no apoio matricial.

\section{Métodos}

O cenário da pesquisa se dá no município de Sobral, Ceará. A inserção do profissional de Educação Física nesse cenário ocorreu antes mesmo da criação do NASF, através da inclusão da categoria na segunda turma da Residência Multiprofissional em Saúde da Família no ano $2000^{9}$. O município tradicionalmente estruturou seu modelo de atenção à saúde adotando uma proposta de rede que acolhe a formação de profissionais e preocupa-se com a educação permanente em saúde (sistema saúde-escola ou docente-assistencial), bem como é sensível à operacionalização de tecnologias como o apoio matricial ${ }^{10}$.

A metodologia qualitativa de pesquisa se mostrou pertinente na medida em que o objetivo pressupõe a empiria sem delimitação prévia de desfechos, com abertura ao novo. Nesse sentido, houve escolha intencional dos participantes. Foram convidados para participar profissionais de Educação Física que possuíam relevante aproximação com o objeto desse estudo, quais sejam: ter atuado na assistência - APS - por no mínimo seis meses; ter atuado no âmbito da formação voltado para a APS (professores universitários e/ou corpo docente do programa de residência); na pesquisa (professores ou alunos de pós-graduação); e/ou na gestão (gerência de Unidades Básicas de Saúde, ou cargos na Secretaria Municipal de Saúde). Excluindo aqueles que estavam, de algum modo, afastados da prática profissional, constituíram-se como participantes da pesquisa 11 profissionais de Educação Física, sendo alguns deles com funções cumulativas, categorizando-se como: quatro envolvidos com ensino, três, com pesquisa, oito, com a assistência e dois, na gestão.

A construção dos dados empíricos se deu a partir de técnicas de Grupo focal e da observação da prática profissional, tendo o diário de campo como instrumento. A síntese da construção dos dados empíricos pode ser vislumbrada no Quadro 1.

A sistematização das informações, tanto em relação às temáticas provisórias discutidas no Grupo focal 3 quanto àquelas referente ao material empírico final, deu-se com base na análise de conteúdo do tipo análise temática ${ }^{11}$. Sendo assim, seguimos as etapas de pré-análise, exploração do material e tratamento dos resultados obtidos, com ênfase na elaboração de convergências, complementariedades e divergências entre o material empírico produzido. Esse processo resultou em duas categorias, constituídas por núcleos de sentido, conforme sintetizado na Figura 1.

Quadro 1 - Síntese da construção da empiria do estudo, Sobral, Ceará, 2018.

\begin{tabular}{lll}
\hline Encontro & Descrição do momento & Temáticas trabalhadas \\
\hline Grupo focal 1 & Participação de 10 sujeitos de duração de 85 minutos. & $\begin{array}{l}\text { Entendimento de apoio matricial, ações desenvolvidas nessa perspectiva e } \\
\text { desafios/dificuldades para operacionalização de tais ações. }\end{array}$ \\
$\begin{array}{lll}\text { Grupo focal } 2 & \text { Participação de nove sujeitos e duração de } 84 \text { minutos. } & \text { Competências para o trabalho com apoio matricial. Pactuação de cronograma } \\
\text { para observação participante. }\end{array}$ \\
$\begin{array}{lll}\text { Observação } & \text { Observação não sistemática, com registro em diário de } \\
\text { campo. }\end{array}$ & $\begin{array}{l}\text { Confronto entre a discussão apresentada nos grupos focais um e dois e os } \\
\text { cenários de prática. }\end{array}$ \\
Grupo focal 3 & Participação de nove sujeitos e duração de 70 minutos. & $\begin{array}{l}\text { Discussão, análise coletiva e reformulação das temáticas provisórias constituídas a } \\
\text { partir dos encontros de grupo focal anteriores e observação participante. }\end{array}$ \\
\hline
\end{tabular}


Estas são apresentadas separadamente enquanto resultados, cuja discussão é explorada na sequência.

\begin{tabular}{|l|l|}
\hline \multirow{2}{*}{ Trabalho no contexto } & $\begin{array}{l}\text { • Rede Cegonha } \\
\text { • Rede de Atenção à Saúde da } \\
\text { Mulher }\end{array}$ \\
das Redes de & - Rede de Atenção Psicossocial \\
Atenção à Saúde & $\begin{array}{l}\text { - Rede de Atenção às Doenças } \\
\text { e Condições Crônicas } \\
\text { - Rede de Cuidado à Pessoa com } \\
\text { Deficiência }\end{array}$ \\
\hline
\end{tabular}

\begin{tabular}{|c|c|}
\hline $\begin{array}{l}\text { Possibilidades de } \\
\text { intervenções em } \\
\text { saúde }\end{array}$ & $\begin{array}{l}\text { - Apoio Matricial Intersetorial } \\
\text { - Acolhimento } \\
\text { - Projeto Terapêutico Singular } \\
\text { - Interconsulta } \\
\text { - Intervisita } \\
\text { - Apoio a Unidades Básicas de Saúde } \\
\text { - Apoio a grupos de práticas corporais }\end{array}$ \\
\hline
\end{tabular}

Figura 1 - Categorias empíricas e respectivos núcleos de sentido emergentes, Sobral, Ceará, 2018.

Os procedimentos empregados no estudo foram aprovados pelo Comitê de Ética em Pesquisa da Universidade Estadual Vale do Acaraú, com parecer número 470.565, tendo sido obtido o Termo de Consen- timento Livre e Esclarecido - TCLE.

\section{Resultados}

Traballho no contexto das Redes de Atenção à Saúde Entre os dados produzidos ao longo da pesquisa, evidenciou-se a preocupação em destacar o direcionamento das intervenções, ou públicos que os profissionais consideram contemplar através de ações de apoio matricial. A sistematização realizada evidencia que as temáticas agrupadas possuíam associação direta com as Redes de Atenção à Saúde e com os seus respectivos equipamentos sociais (como hospitais, maternidades, CRAS, entre outros).

Entende-se por RAS "arranjos organizativos de ações e serviços de saúde, de diferentes densidades tecnológicas que, integradas por meio de sistemas de apoio técnico, logístico e de gestão, buscam garantir a integralidade do cuidado" ${ }^{12}$. Para implantação das RAS, o Ministério da Saúde, após pactuação tripartite em 2011, priorizou cinco redes temáticas: rede cegonha, rede de atenção à urgência e emergência, rede de atenção psicossocial, rede de atenção às doenças e con-

Quadro 2 - Categorias e falas que abordam as RAS articuladas pelo profissional de Educação Física.

Rede cegonha: Pré-natal, puérperas

No pré-natal, perceber junto com a equipe, por exemplo, com a enfermeira, perceber que dificuldades elas tinham naquele momento de pré-natal. E aí, possivelmente, depois criar espaços de discussão para perceber como que poderia potencializar esse pré-natal, e aí surgiu daí a ideia de reconstruir o grupo de gestantes que tinha no território, mas que estava desativado e a gente conseguiu conversando com a enfermeira e ela disse que, em algumas áreas de risco, elas têm dificuldades de vincular com essas gestantes e até mesmo de participar do pré-natal (Participante 1, Grupo focal 1).

[...] na Puericultura [...], além de conhecer para além dos estágios de desenvolvimento do ser humano, saber com quantos meses os reflexos são alterados ou até as questões motoras da criança é de ver que as crianças estão chegando para um atendimento na puericultura e estão chorando muito e a gente pode deixar aquele ambiente mais humanizado. A gente usa muitos recursos, como vídeos, pintura e até bola. A gente usa esse instrumento do brincar para realmente olhar esses estágios de desenvolvimento (Participante 7, Grupo focal 2).

Rede de atenção à saúde da mulher: planejamento familiar e prevenção

Eu acredito que no planejamento familiar o mais importante é a noção de corpo, o reconhecimento de qual planejamento ela quer e que alterações esse planejamento vai gerar com corpo dela, ingestão de drogas. Acho que para o apoio matricial, nesse momento, o professor de Educação Física deve ter uma visão, do meu ponto de vista, seria que uma visão do corpo, que droga ela vai estar colocando dentro do corpo dela, essa relação com a promoção da saúde dela com a atividade física, qual a postura dela em relação a determinado de tipo de remédio, de anticoncepcional, aquele outro, que alterações podem ocorrer no ciclo dela, no rendimento dela enquanto na academia que ela quer fazer, no dia a dia (Participante 10, Grupo focal 2).

Rede de Atenção Psicossocial: usuários em sofrimento psíquico

A escuta e a visão ampliada de que aquele momento que às vezes é um momento que a pessoa tá vivendo, ela não consegue dormir, ela está com insônia, ela está com muitos problemas em casa e isso está gerando essa insônia, e às vezes ela só quer ser escutada, ela quer dormir, mas ela acha que pra ela dormir, ela precisa do remédio, e normalmente psiquiatra prescreve logo o remédio para ela dormir, porque ela precisa dormir. Porém, às vezes, o diálogo e a conversa resolvem. Já aconteceu muitas vezes a gente agendar uma terapeuta ocupacional, um educador físico, faz uma visita e não tem a necessidade de encaminhar de fato para o psiquiatra [...]. Atualmente, a visão da nossa categoria tem sido ampliada muito e nos chamam mais para o Projeto Terapêutico Singular e Matriciamento (Participante 6, Grupo focal 2).

Rede de Atenção às Doenças e Condições Crônicas: hipertensos e diabéticos

A gente teve uma experiência bem interessante, que foi o apoio à equipe de referência, às pessoas com hipertensão e diabetes. Essa experiência de reorganização dessa assistência das pessoas com hipertensão e diabetes provocou todo um realinhamento da organização do serviço, no sentido de implantar processos que não existia antes. Por exemplo, a gente fez uma ficha de classificação de risco, que ajudou a compreender o perfil dessa população. Ao passo em que as pessoas que tinham mais agravos, que tinham mais complicações, foram demandados para o atendimento coletivo multiprofissional (Participante 4 , Grupo focal 1).

Rede de Cuidado à Pessoa com Deficiência: reabilitação

Outra coisa que eu também senti falta, que eu considero que nós temos muito a contribuir, é na reabilitação, reabilitação motora [...] (Participante 11, Grupo focal 2). 
dições crônicas e rede de cuidado à pessoa com deficiência. Isso não impede a existência de outras redes de atenção no município, como rede de atenção à saúde do idoso, do homem, LGBT; como também a inexistência de alguma dessas redes em determinado município.

As falas, cujas categorias são apresentadas no Quadro 2, evidenciam o direcionamento da intervenção e suas respectivas RAS, já com alguns vestígios de desdobramentos de intervenções a serem realizadas.

Assim, o direcionamento da intervenção em que os profissionais de Educação Física desenvolvem apoio matricial são apresentados em forma de síntese no Quadro 3, evidenciando sua relação com as respectivas RAS.

Quadro 3 - Direcionamento da intervenção das práticas de apoio matricial e sua relação com as RAS.

\begin{tabular}{ll}
\hline \multicolumn{1}{c}{ Redes de Atenção à Saúde } & \multicolumn{1}{c}{ Públicos } \\
\hline Rede Cegonha & Pré-natal \\
& Puérperas \\
& Bebês (0-2 anos) \\
& Planejamento Familiar \\
Rede de Atenção à Saúde da Mulher & Prevenção \\
& Usuários em sofrimento \\
Rede de Atenção Psicossocial & psíquico \\
Rede de Atenção às Doenças e Condições & Hipertensos \\
Crônicas & Diabéticos \\
Rede de Cuidado à Pessoa com Deficiência & Reabilitação \\
\hline
\end{tabular}

Tecnologias/práticas do profissional de Educação Física na APS

Para intervir com esses públicos, inseridos em uma estrutura operacional de RAS, os profissionais relatam o uso de uma série de práticas atreladas à realização do apoio matricial. A seguir, são apresentadas definições e narrativas representativas de cada uma delas.

Apoio matricial intersetorial - práticas de apoio matricial desenvolvidas pelo profissional de Educação Física, em que a equipe de referência é de outros setores, geralmente dos setores de assistência social e educação.

[...] a gente tem a questão do trabalho intersetorial como um apoio matricial também, o apoio matricial que se dá na questão intersetorial. A gente consegue construir muito mais que outras categorias, a gente tem esse viés de trabalho na comunidade e tudo mais. Temos que aproveitar essa aproximação com outros locais, como o CRAS, CREAS, time de futebol, para prestar um apoio matricial, puxando para equipe de referência, aproximando comunidade e equipe de referência. Isso também é apoio matricial (Participante 5, Grupo focal 1).

Sujeito 10: Nessa questão do apoio matricial e intersetorial em escolas, partimos do processo que realmente paulofreiriano, que tem um conhecimento lá; então, pra você chegar, fazer um apoio matricial em outro local, é necessário você conhecer o conhecimento dos outros para que você possa realmente trabalhar naquele ambiente de forma mais segura. (Participante 10, Grupo focal 2).

Acolhimento - apesar de o acolhimento ser compreendido como prática transversal a todas as outras, os serviços de saúde na realidade do estudo são organizados de tal forma que há espaço especificamente para essa prática (uma ressignificação da tradicional triagem, no primeiro contato com o usuário). Assim, os profissionais de Educação Física referem como têm atuado nesse cenário.

No espaço do acolhimento, eu adentrei inicialmente para observar: se realmente estava sendo colocado ali a questão da humanização, e aí junto com a equipe a gente estar discutindo como vem sendo o acolhimento da unidade [...]. A ideia que a gente percebia é que era um espaço que as pessoas chegavam e recebiam aquele primeiro atendimento e eram encaminhadas, mas será que a ideia do acolhimento em si acontecia? E aí a gente pensou em levar esse momento para discutir com a equipe, em roda [reunião], o que a gente entende por acolhimento. $\mathrm{E}$ aí, depois, minimamente tentar inserir algo novo e reconstruir aquele acolhimento e não chegar e dizer que o acolhimento estava errado, mas reconstruir, junto com aquela equipe, o que é o acolhimento, realmente (Participante 1, Grupo focal 1).

[...] isso vai para a humanização de o cuidado, para o ser, e aí $o$ acolhimento também. A gente precisa fazer uma escuta mais humanizada, porque os usuários chegam com uma demanda e gente acaba terminando sempre com outra, como acontece nos territórios. (Participante 7, Grupo focal 2).

Projeto terapêutico singular (PTS) - trata-se de uma tecnologia eminentemente trans/interdisciplinar, que enfatiza a participação do usuário na construção de seu projeto terapêutico. Uma possibilidade de sua organização é com adaptações a cinco etapas: avaliação da situação, definição de hipóteses diagnósticas, definição de metas, divisão de responsabilidades e reavaliação ${ }^{13}$. Nesse aspecto, os profissionais destacam as principais dificuldades para sua operacionalização.

A gente precisa de ter uma corresponsabilidade diante dos processos, então, assim dizendo, alguns processos de apoio desenvolvido, o que mais se fez na unidade de saúde foi o PTS [...]. Muitas vezes, acham que naquele caso $\mathrm{X}$ não temos a contribuir, mas temos muito, caso a gente começar a sair da nossa caixinha, que a gente mesmo se coloca. Para muitos, a categoria é só "prática corporal, pronto e acabou" [ironiza]. (Participante 5, Grupo focal 1).

Um desafio bem prático do PTS começa pelas agendas. Como vão conseguir sentar todos de forma sistemática a longo prazo? Requer uma sistemática a longo prazo. (Participante 5, Grupo focal 1).

Interconsulta - trata-se de um espaço da clínica individual, mas com a presença e o trabalho integra- 
do com profissionais de diferentes categorias. Nesse caso, destacamos que esse compartilhamento ocorre por vezes em agendas específicas das outras categorias (em que o profissional de Educação Física atua como apoiador matricial), e em outras, menos recorrentes, em “consultas" da Educação Física.

A interconsulta, a gente faz muito, principalmente com a nutrição e com a enfermagem [...]. Eles entendem que é importante fazer caminhada, mas não querem que a pessoa simplesmente coloque um chinelo ou um tênis e saia andando, e sim uma atividade que seja direcionada, bem orientanda (Participante 11, Grupo focal 2).

No campo da interconsulta e intervisita, uma coisa que a gente precisa saber fazer e fazer com ética é a questão dos prontuários, porque existem limitações na formação inicial, como ter segurança em evoluir prontuários. (Participante 4, Grupo focal 2).

Intervisita - tem por base o mesmo caráter da interconsulta, com a singularidade de ocorrer em visitas domiciliares.

A intervisita é uma visita domiciliar que conta com presença de vários profissionais, geralmente o ACS e outros profissionais que mais têm relação com aquele caso, com aquela demanda. (Participante 1, Grupo focal 1)

Como em algumas rodas de categoria, e também nas visitas, que é muito rica, tanto com a enfermagem, quando com nutrição e psicologia, a gente pode estar atuando com o apoio matricial. (Participante 8, Grupo focal 1)

Apoio a unidades básicas de saúde - trata-se de intervenções pontuais, realizadas em unidades básicas de saúde que não possuem profissionais de Educação Física. Em discussões permeadas por reflexões que resgatam a precarização do trabalho, os profissionais relatam parcerias realizadas com equipes de NASF sem Educação Física.

Enquanto práticas corporais, eu vejo o apoio matricial da Educação Física como uma necessidade para as unidades que não possuem o profissional. Muitas vezes, eu recebia esse convite, como eu sei que outros colegas também recebiam. A gente pode estar facilitando e também ajudando, porque o repertório daquele profissional já se esgotou e ele precisa de coisas novas, de atividades novas, e até de uma pessoa nova [risos]. Assim, já facilitei alguns grupos, já ajudei alguns outros profissionais, a gente já trocou figurinhas. (Participante 7, Grupo focal 1).

No momento, eu atendo quase especificamente só em uma área, que facilita algumas ações que são desenvolvidas, mas também dou apoio a duas unidades, próximo a minha área, e uma delas não tem o profissional de Educação Física. (Participante 9, Grupo focal 1).
Apoio a grupos de práticas corporais cuidados por líderes comunitários ou por agentes comunitários de saúde (ACS) - trata-se de uma discussão importante levantada pelos participantes, que se posicionam e atuam no sentido de reconhecer, fortalecer e incentivar o desenvolvimento dessa prática também por outras lideranças.

Dentro da fala da Participante 6, na época da minha residência, a gente fazia também esse apoio matricial com o ACS, dando suporte a eles para que desenvolvam algumas atividades sem a presença do profissional de Educação Física. (Participante 2, Grupo focal 1).

[...] existe um grupo hoje de caminhada no [nome suprimido] que já existe há 13 anos. Entra profissional, sai profissional, e o grupo está lá, firme e forte, independente do profissional ou não. Dentro do meu fazer no centro de saúde, tento conversar com a pessoa que está mais à frente, a treinadora [risos], tentando explicar um pouquinho mais a questão do movimento (Participante 3, Grupo focal 1).

Considerando os aspectos apresentados na categoria "Trabalho no contexto das Redes de Atenção à Saúde", bem como os aqui apresentados - "Tecnologias/ práticas do profissional de Educação Física no NASF”, constituída por apoio matricial intersetorial, acolhimento, construção compartilhada de plano terapêutico singular, interconsulta, intervisita, apoio a unidades básicas de saúde e apoio a grupos de práticas corporais cuidados por líderes comunitários ou por ACS -, passamos a apresentar a discussão.

\section{Discussão}

Há alguns estudos que apontam para a necessidade de considerar o contexto em que o usuário vive para pensar, de modo dialogado, seu plano de cuidado ${ }^{14-16}$. Os resultados do presente estudo, além de reafirmar a necessidade de pensar a organização dos processos de trabalho centrada no usuário, traz um conjunto de elementos que dizem respeito à relevância de considerar que cada usuário está vinculado a alguma(s) RAS. Essa premissa implica uma compreensão sistêmica em relação a como o SUS se organiza. A complexidade apresentada pelo presente estudo se apresenta de pelo menos dois pontos de vista: setorial e intersetorial.

Setorial, partindo do entendimento de que a APS é coordenadora e ordenadora das RAS. Sendo assim, um usuário que é atendido (pontualmente ou não) em outro nível de atenção (como no Centro de Atenção Psicossocial - CAPS, aproveitando a saúde mental como exemplo), necessariamente continua vinculado, longitudinalmente, à APS. Além disso, o profissional da APS precisa 
estar ciente de que pode articular, caso necessário, para que algum usuário por ele acompanhado seja atendido por essa unidade de referência (CAPS, no exemplo em questão). A mesma lógica se aplica a outras RAS (não somente a Rede de Atenção Psicossocial apresentada). Vale ressaltar que as redes são integradas por vários "nós", ou seja, não se constituem em planos paralelos.

Intersetorial, partindo do entendimento de que a saúde, entendida não somente como ausência de doenças (em sentido restrito), ou como completo bem-estar (em sentido idealizado), constitui-se pelos determinantes sociais da saúde (tais como alimentação, moradia, lazer, atividade física, segurança...) e, portanto, não pode ser alcançada sem articulação com outros setores. Os resultados revelam, por exemplo, articulação na forma de apoio matricial entre o profissional de Educação Física com o setor da assistência social, o Centro de Referência de Assistência Social - CRAS.

Diante disso, a originalidade do presente estudo ao abordar, inicialmente, o trabalho no contexto das RAS, implica o entendimento de que cada usuário atendido pelo profissional de Educação Física compõe uma rede complexa. Apesar de não ter sido ênfase deste estudo, na perspectiva do cuidado centrado no usuário, além da noção sistêmica de que a Educação Física integra um serviço de saúde localizado em um projeto setorial maior (rede setorial, particularmente o setor saúde) e pode realizar articulações intersetoriais (rede intersetorial), a singularidade de cada usuário aponta para redes sociais de apoio. Nesse momento, no que se refere a essa "terceira via", entendemos como redes sociais de apoio aquelas às quais o usuário se vincula que não necessariamente compõem as duas já apresentadas. No caso das práticas corporais, por exemplo, o usuário pode ter forte vínculo com um grupo de futebol presente na comunidade, não vinculado às duas redes institucionalizadas apresentadas (setorial e intersetorial).

O genograma e o ecomapa são instrumentos relevantes nesse sentido, sendo pertinente serem conhecidos e trabalhados pela Educação Física. Trata-se de uma representação gráfica da rede social de apoio, bem como dos conflitos em potencial, tanto na composição familiar (genograma) quanto na relação entre usuário e comunidade (ecomapa) ${ }^{17-18}$.

O entendimento do contexto do usuário e da organização dos serviços de saúde do Brasil se faz pertinente na medida em que transpor saberes e práticas da categoria já instituídas a priori, para o âmbito do SUS, como programas de atividades físicas/práticas corpo- rais, que desconsideram essa noção sistêmica, é problemático. Um debate semelhante na área ocorreu ao tratar da disciplina Educação Física escolar, particularmente sobre o esporte, em que se constituiu a perspectiva de um esporte "na" escola, caracterizada por uma relativa descontextualização com a organização política e institucional da educação brasileira, para um esporte "da" escola. Com argumentação semelhante, entendemos que é preciso pensar uma Educação Física "do" SUS.

Uma ressignificação da Educação Física, constituindo uma prática "do" SUS, implica pensar que sua atuação isolada não é suficiente. Como possibilidade, o apoio matricial revela uma articulação deste (o apoiador, como especialista) com a equipe de referência (como cuidador longitudinal), envolve um trabalho interprofissional. Perpassa ainda por um trabalho pedagógico-formativo, na medida em que o profissional de Educação Física apoia pedagogicamente a equipe de referência, bem como aprende com esta ${ }^{7}$. Por fim, configura-se como um trabalho usuário-centrado, por ser balizado, necessariamente, por suas demandas singulares. Estes três elementos podem ser considerados premissas para mobilização das práticas de apoio matricial nas tecnologias relatadas pelos profissionais: apoio matricial intersetorial, acolhimento, PTS, interconsulta, intervisita, apoio a unidades básicas de Saúde e apoio a grupos de práticas corporais cuidados por líderes comunitários ou por ACSs. Assim, pode-se dizer importante um conjunto de atitudes, quais sejam: ser um profissional aprendente, promover a clínica ampliada, conhecer a rede, reorganizar o cuidado em saúde e participar dos espaços políticos ${ }^{8}$.

Analisado superficialmente, isso pode induzir que o profissional de Educação Física, ao desenvolver apoio matricial, não trabalha com atividades físicas/práticas corporais. Não se trata disso! As atividades físicas/práticas corporais se fazem presentes de modo transversal às práticas do profissional de Educação Física, no desenvolvimento de qualquer tecnologia relatada pelos profissionais. Passam, porém, por uma ressignificação: são tratadas de modo dialógico-pedagógico e não prescritivo; construídas/produzidas e não transmitidas. Por vezes, inclusive, é preciso assumir outras funções, não diretamente ligadas à especificidade da área, sobretudo quando o profissional de referência para determinado caso é de outra categoria profissional.

Nesse sentido, é preciso reconhecer que as tecnologias apresentadas como práticas de apoio matricial não se constituem como modelos a serem acriticamen- 
te desenvolvidos. Cada caso demanda acolhimento e articulação coerente com sua respectiva singularidade. Além disso, o presente estudo teve a pretensão de apresentar uma perspectiva panorâmica das possibilidades de intervenção, as quais, individualmente, demandam estudo específico em profundidade.

Ainda considerando a potência da experiência significativa do trabalho desenvolvido no cenário deste estudo - Sobral, Ceará -, é preciso ponderar que não necessariamente as Redes de Atenção à Saúde estruturadas pelos municípios condizem com as redes prioritárias propostas pelo Ministério da Saúde; a proposta municipal deve estar adequada às necessidades locais e não verticalmente determinada. Assim, cada profissional de Educação Física vinculado à APS precisa consultar dados institucionais do município em que atua para que possa saber quais redes se encontram instituídas e as respectivas unidades de referências de cada rede. Além disso, cabe ressaltar que os participantes do estudo não mencionam o direcionamento da intervenção para alguns públicos, o que remete a pensar sua invisibilidade, ainda que se constituam Políticas Nacionais de Saúde próprias, tais como: lésbicas, gays, bissexuais e transexuais (LGBT), saúde integral do homem, povos indígenas e população negra.

Diante do exposto, conclui-se que a complexidade dos cenários e as práticas de apoio matricial da Educação Física na APS, no contexto das RAS, revelam a limitação de se elaborar um conjunto de protocolos/ procedimentos que se enquadrem à condição patológica do usuário em variáveis predeterminadas (de tempo e intensidade, por exemplo) ou restrita à prescrição de atividade física. Ao mesmo tempo, apontam para a necessidade de se considerar a estrutura operacional das redes (setorial, intersetorial e social de apoio), bem como tecnologias/práticas centradas no trabalho interprofissional, pedagógico-formativo e centrado no usuário. O trabalho na APS tem como premissa o entendimento da organização dos serviços de saúde, além das especificidades da categoria profissional.

\section{Conflito de interesse}

Os autores declaram não haver conflito de interesses.

\section{Contribuição dos autores}

Oliveira BN e Wachs F, contribuíram com a concepção do manuscrito, análise e interpretação dos dados, redação do manuscrito e revisão crítica relevante do conteúdo intelectual. Ambos aprovaram a versão a ser publicada.

\section{Agradecimento}

Agradecemos ao Ministério da Saúde pelo apoio com uma bolsa de Residência Multiprofissional em Saúde.

\section{Referências}

1. Ministério da Saúde. Portaria n.2436, de 21 de setembro de 2017. Aprova a Política Nacional de Atenção Básica, estabelecendo a revisão de diretrizes para a organização da Atenção Básica, no âmbito do Sistema Único de Saúde (SUS). In: Diário Oficial da União [online], 2017. [citado em 2019 mar 19]. Disponível em: http://www.in.gov.br/ materia/-/asset_publisher/Kujrw0TZC2Mb/content/ id/19308123/do1-2017-09-22-portaria-n-2-436-de-21-desetembro-de-2017-19308031

2. Ministério da Saúde. Política Nacional de Atenção Básica. Brasília: Ministério da Saúde, 2012.

3. Ceccim RB, Bilibio LF. Singularidades da Educação Física na saúde: desafios à educação de seus profissionais e ao matriciamento interprofissional. In: Fraga AB, Wachs F. (eds). Educação Física e Saúde Coletiva: políticas de formação e perspectivas de intervenção. Porto Alegre: UFRGS; 2007.p. 47-62.

4. Santos S, Benedetti TRB. Cenário de implantação do Núcleo de Apoio à Saúde da Família e a inserção do profissional de Educação Física. Rev Bras Ativ Fis Saúde. 2012;17(3):188-94.

5. Scabar TG, Pelicioni AF, Pelicioni MCF. Atuação do profissional de Educação Física no Sistema Único de Saúde: uma análise a partir da Política Nacional de Promoção da Saúde e das Diretrizes do Núcleo de Apoio à Saúde da Família-NASF. J Health Sci Inst. 2012;30(4):411-8.

6. Santos S, Benedetti T, Sousa T, Fonseca S. Apoio Matricial e a atuação do Profissional de Educação Física do Núcleo de Apoio à Saúde da Família. Rev Bras Ativ Fis Saúde. 2017;22(1):54-65.

7. Oliveira BN, Wachs F. Educação Física e Atenção Primária à Saúde: apropriações acerca do apoio matricial. Movimento (Porto Alegre). 2018;24(1):173-86.

8. Oliveira BN, Wachs F. Educação física, atenção primária saúde e organização do trabalho com apoio matricial. Rev. Bras. Ciênc. Esporte. 2019, 41(2):183-89.

9. Silva ALF, Oliveira BN. A trajetória da Educação Física no SUS em Sobral-CE: um resgate histórico. Conexões: Educ. Fís., Esporte e Saúde. 2013;11(2):193-207.

10. Souza MLT. Apoio matricial em saúde mental: uma proposta de educação permanente em saúde? Sanare (Sobral, Online). 2011;10(2):28-33.

11. Minayo MCS. O desafio do conhecimento: pesquisa qualitativa em saúde. 11. ed. São Paulo: Hucitec, 2008.

12. Ministério da Saúde. Portaria no 4.279 , de 30 de dezembro de 2010. Estabelece diretrizes para a organização da Rede de Atenção à Saúde no âmbito do Sistema Único de Saúde (SUS). Brasília: Ministério da Saúde, 2010.

13. Miranda FAC, Coelho EBS, Moré CLOO. Projeto terapêutico singular. Florianópolis: Universidade Federal de Santa Catarina, 2012.

14. Motta BFB, Perucchi J, Filgueiras MST. O acolhimento em Saúde no Brasil: uma revisão sistemática de literatura sobre o tema. Rev. SBPH. 2014;17(1):121-39.

15. Agreli HF, Peduzzi M, Silva MC. Atenção centrada no paciente na prática interprofissional colaborativa. Interface (Botucatu). 2016;20(59):905-16. 
16. Oliveira JSB, Suto CSS, Silva RS. Tecnologias leves como práticas de enfermagem na atenção básica. Revista Saúde Com. 2016;12(3):613-21.

17. Pennafort VPS, Queiroz MVO, Nascimento LC, Guedes MVC. Rede e apoio social no cuidado familiar da criança com diabetes. Rev Bras Enferm. 2016;69(5):912-9.
18. Nascimento LC, Rocha SMM, Hayes VE. Contribuições do genograma e do ecomapa para o estudo de famílias em enfermagem pediátrica. Texto \& Contexto Enferm. $2005 ; 14(2): 280-6$.

Recebido: 19/03/2019

Aprovado: 02/07/2019

\section{Como citar este artigo:}

Oliveira BN, Wachs F. Educação Física e Atenção Primária à Saúde: o apoio matricial no contexto das redes. Rev Bras Ativ Fis Saúde. 2018;23:e0064. DOI: 10.12820/rbafs.23e0064 